\title{
Real-Space Observation of Magnon Interaction with Driven Space-Time Crystals
}

\author{
Nick Träger@ ${ }^{1,}{ }^{*}$ Paweł Gruszecki, ${ }^{2}$ Filip Lisiecki, ${ }^{3}$ Felix Groß, ${ }^{1}$ Johannes Förster, ${ }^{1}$ Markus Weigand,${ }^{1,4}$ Hubert Głowiński, ${ }^{3}$ \\ Piotr Kuświk $\odot,{ }^{3}$ Janusz Dubowik $\odot,{ }^{3}$ Gisela Schütz, ${ }^{1}$ Maciej Krawczyk ${ }^{2},{ }^{2}$ and Joachim Gräfe $\oplus^{1, \dagger}$ \\ ${ }^{1}$ Max Planck Institute for Intelligent Systems, Heisenbergstr. 3, 70569 Stuttgart, Germany \\ ${ }^{2}$ Adam Mickiewicz University, Faculty of Physics, ul. Uniwersytetu Poznańskiego 2, 61-614 Poznań, Poland \\ ${ }^{3}$ Institute of Molecular Physics, Polish Academy of Sciences, Mariana Smoluchowskiego 17, 60-179 Poznań, Poland \\ ${ }^{4}$ Helmholtz-Zentrum Berlin für Materialien und Energie, Albert-Einstein-Str. 15, 12489 Berlin, Germany
}

(Received 17 March 2020; revised 14 August 2020; accepted 9 December 2020; published 3 February 2021)

\begin{abstract}
The concept of space-time crystals (STC), i.e., translational symmetry breaking in time and space, was recently proposed and experimentally demonstrated for quantum systems. Here, we transfer this concept to magnons and experimentally demonstrate a driven STC at room temperature. The STC is realized by strong homogeneous microwave pumping of a micron-sized permalloy (Py) stripe and is directly imaged by scanning transmission $\mathrm{x}$-ray microscopy (STXM). For a fundamental understanding of the formation of the STC, micromagnetic simulations are carefully adapted to model the experimental findings. Beyond the mere generation of a STC, we observe the formation of a magnonic band structure due to back folding of modes at the STC's Brillouin zone boundaries. We show interactions of magnons with the STC that appear as lattice scattering, which results in the generation of ultrashort spin waves (SW) down to 100-nm wavelengths that cannot be described by classical dispersion relations for linear SW excitation. We expect that room-temperature STCs will be useful to investigate nonlinear wave physics, as they can be easily generated and manipulated to control their spatial and temporal band structures.
\end{abstract}

DOI: 10.1103/PhysRevLett.126.057201

Magnons, which are the quanta of SWs, were intensely discussed in the past decade, revealing new fundamental phenomena in magnetism [1-4]. Especially intriguing is the analogy to other bosonic quanta, like photons and the corresponding photonics applications. From a fundamental point of view, emerging quantum phenomena are of great interest. Here, artificial magnonic crystals, i.e., systems with periodically modulated magnetic properties, are especially alluring as they allow one to generate and manipulate SW band structures [4-7].

Recently, the concept of periodic modulation was extended from space into time, leading to the idea of a time crystal by Wilczek [8]. A review of the physics of these time crystals can be found in Ref. [9]. Watanabe and Oshikawa already noticed that the existence of time crystals should be a logical consequence of Lorentz-invariant space-time and long-range order in spatial directions [10]. Indeed, the definition of a time crystalline structure is deduced from ordinary space crystals. The most important criterion for formation of a crystal is the breaking of

Published by the American Physical Society under the terms of the Creative Commons Attribution 4.0 International license. Further distribution of this work must maintain attribution to the author(s) and the published article's title, journal citation, and DOI. Open access publication funded by the Max Planck Society. continuous spatial translation symmetry into a discrete translation symmetry. Equivalently, a time translation symmetry break (TTSB) is essential for time crystals. However, quantum equilibrium states have time-independent observables that basically forbid TTSB in the ground state [11]. Only with long-range interactions was a Hamiltonian found to bypass the no-go theorem of Watanabe and Oshikawa. Thus, time crystalline phases can be generated within closed quantum systems, showing resilience to local perturbations [12]. Yet, nonequilibrium states, like periodically driven many-body Floquet systems, can also possess a time translation symmetry governed by the external frequency input $[13,14]$. Several experiments confirmed a TTSB in quantum Floquet systems, revealing observables with subharmonic responses [15-17].

The combination of both of these symmetry breakings defines a so-called space-time crystal that exhibits periodicity in space and time. This combination was realized by Smits et al. as a direct observation of space-time crystallinity in a superfluid quantum gas [18]. Additionally, Kreil et al. recently proposed a STC at room temperature in a Bose-Einstein condensate (BEC) of magnons [19]. However, these experiments were limited to quantum systems and only showed the general existence of STCs.

In this work, we unite the fundamental STC concept within the quantum regime with the world of magnonics and present an exceptional case for nonlinear wave physics in a comparatively large structure. While the existence of 
STCs has been shown in the literature, lattice scattering processes have not yet been observed [18,19]. To this end, we generate a driven STC in a Py waveguide and directly image it by time-resolved STXM with $\mathrm{x}$-ray circular dichroism (XMCD) contrast (20-nm spatial and 50-ps temporal resolution) $[2,20]$. We use this technique to show the formation of a driven STC and investigate its interaction with magnons at room temperature. Thereby, we observe lattice scattering into higher Brillouin zones and the generation of ultrashort SWs that cannot be explained by conventional dispersion theory. Additionally, we carefully employ micromagnetic simulations to model the experiment and to gain a fundamental understanding of the experimental observations [21] (cf. Supplemental Material (SM) [22]). Time-resolved STXM measurements were performed at the MAXYMUS end station (UE46PGM2 beamline) at the BESSY II synchrotron radiation facility. Further details regarding the experimental realization and setup are described in the SM [22].

The magnonic waveguide consists of a 30-nm-thick, 1.4- $\mu$ m-wide, $11-\mu \mathrm{m}$-long Py $\left(\mathrm{Fe}_{20} \mathrm{Ni}_{80}\right)$ stripe deposited below a coplanar radio frequency (rf) waveguide (copper). Figure 1(a) shows a sketch of the sample with the signal line (shown in dark gray) and the magnonic structure (shown in yellow). A static bias field $B_{\text {ext }}$ is applied along the signal line, and SWs are excited by a continuous wave (cw) rf field. As the signal line is much larger than the magnonic waveguide, the rf field can be considered as uniform. Since the magnetic moments are rotated by

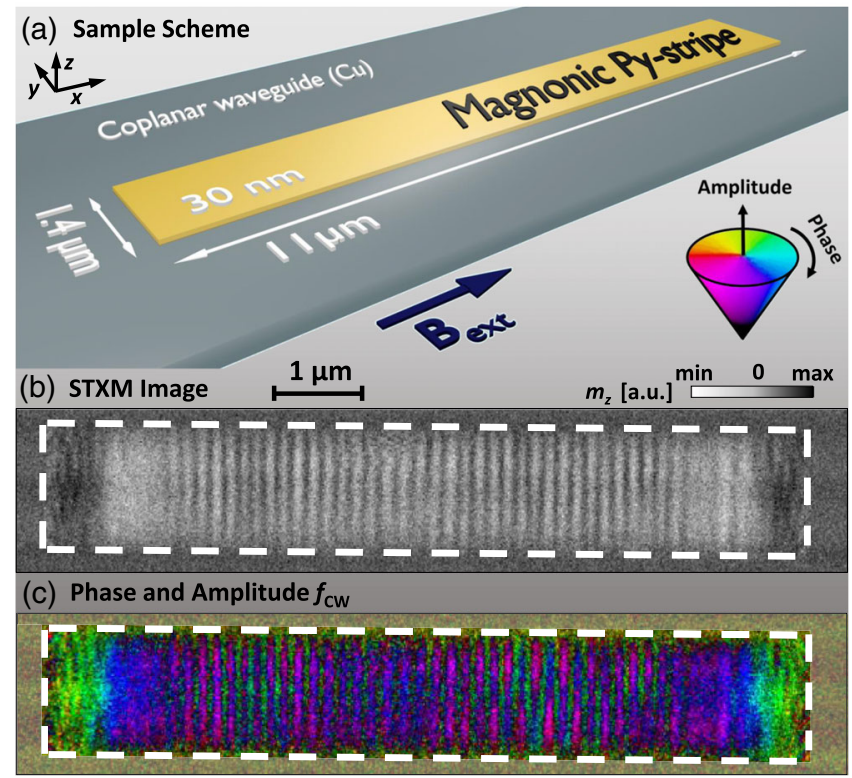

FIG. 1. (a) Sketch of the sample with one magnonic Py stripe (yellow) and a coplanar waveguide (gray). (b) Snapshot of a timeresolved STXM movie. The gray scale represents the $m_{z}$ component. (c) Phase and amplitude map at $f_{\mathrm{cw}}$ after FFT in time through each pixel of the STXM movie. The color code shows the amplitude and phase information. approximately $90^{\circ}$ at the short edges of the stripe (cf. Fig. S1 in SM [22]), SWs are excited by the combination of oscillations of Néel-type domain walls with the nonuniform dynamic demagnetizing field generated by precessing magnetization at the edges [23-25].

Figure 1(b) shows a snapshot of the reduced magnetization component $m_{z}=M_{z} / M_{\mathrm{s}}$ with the saturation magnetization $M_{\mathrm{s}}$ and the $z$ component of the magnetization $M_{z}$ from a time-resolved STXM movie with $\mathrm{cw}$ excitation at $f_{\mathrm{cw}}=$ 4.2 $\mathrm{GHz}$ and an applied field $B_{\text {ext }}=8 \mathrm{mT}$. A periodic SW pattern is clearly visible. For further analysis, we use a temporal FFT algorithm to access the amplitude and relative phase of the SW, which is depicted in Fig. 1(c), where the amplitude is encoded as brightness and the phase as color.

Subsequently, a spatial FFT allows transition into $k$-space, where the wave vector is $k=\lambda^{-1}$. The $k$-space transformation of the SWs in Fig. 1(c) is shown in Fig. 2(c). Further details on the data evaluation procedure can be found elsewhere [26]. Next to the dc peak $\left(k_{x, y}=0 \mu \mathrm{m}^{-1}\right)$ in Fig. 2(c), two additional peaks are observed, representing distinct SW modes. These peaks occur at $k_{1}=5 \mu \mathrm{m}^{-1}$ and $k_{2}=10 \mu \mathrm{m}^{-1}$, corresponding to wavelengths of $\lambda_{1}=200 \mathrm{~nm}$ and $\lambda_{2}=100 \mathrm{~nm}$, respectively. Selective back transformation allows visualization of the real-space

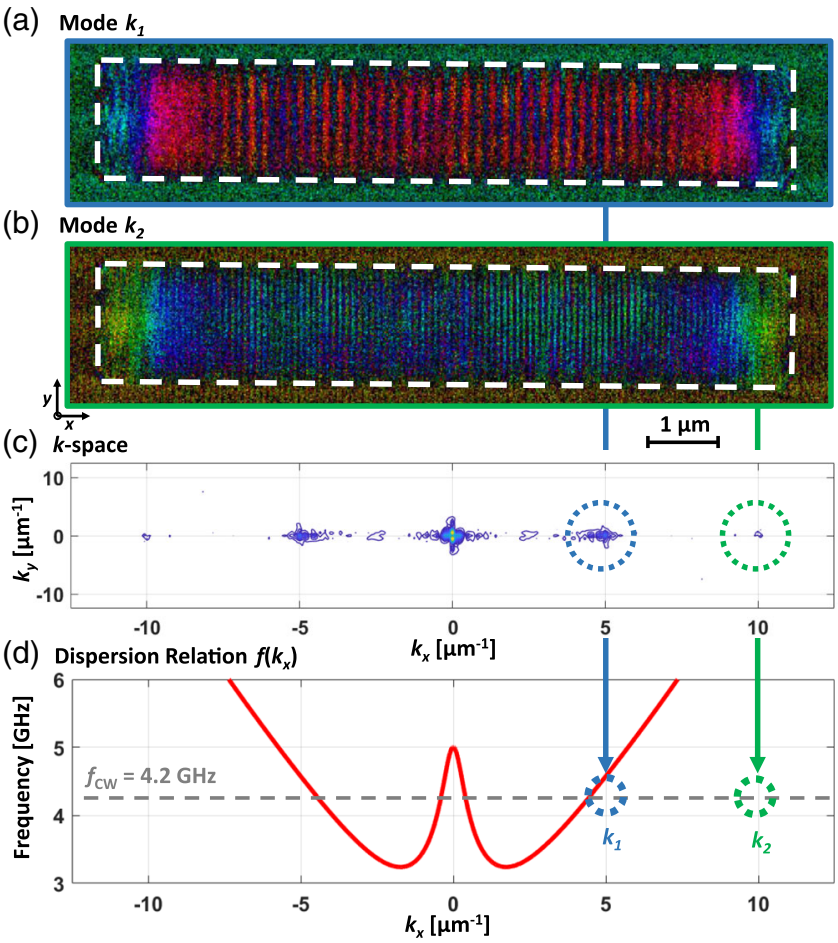

FIG. 2. (a) Phase and amplitude map of $k_{1}$. (b) Phase and amplitude map of $k_{2}$. (c) $k$-space retrieved from the spatial FFT of the phase and amplitude map in Fig. 1(c). Next to the dc peak in the middle, peaks at $k_{1}=5 \mu \mathrm{m}^{-1}$ and $k_{2}=10 \mu \mathrm{m}^{-1}$ can be observed. (d) Dispersion relation $f\left(k_{x}\right)$ (red line) and an excitation frequency $f_{\mathrm{cw}}=4.2 \mathrm{GHz}$. The calculated dispersion describes mode $k_{1}$ (blue arrow) but fails to capture $k_{2}$ (green arrow). 
mode profiles of the individual modes shown in Figs. 2(a) and 2(b), respectively.

SW dispersion theory for infinite films was extended by Guslienko et al. and Brächer et al. to consider lateral confinement in magnonic waveguides [27,28]. Figure 2(d) shows the theoretical dispersion relation for the confined geometry discussed here. The parameters for these calculations were also used for micromagnetic simulations and can be found in the SM [22]. While we find good agreement for the first mode $\left(k_{1}=5 \mu \mathrm{m}^{-1}\right)$, the second mode $\left(k_{2}=10 \mu \mathrm{m}^{-1}\right)$ cannot be described using linear theory and cannot be attributed to higher-order modes (cf. SM [22], Fig. S2 [28,29]). To explain an allowed mode at $k_{2}=10 \mu \mathrm{m}^{-1}$ and its efficient excitation, in the following, we discuss the formation of a magnonic STC and SW scattering at the STC Brillouin zone boundary.

The $\mathrm{cw}$ rf field leads to the formation of a periodic magnetization pattern and essentially realizes a driven Floquet system with TTSB. While the pattern resembles a standing SW at first sight, it cannot be described as such, as all oscillations are spatially in phase as revealed in
Figs. 3(a) and 3(b) in experiment and simulation, respectively (see extended discussions in the SM [22], Figs. S5S7). Figures 3(a) and 3(b) show the evolution of the out-ofplane magnetization component $m_{z}$ over time. The results of the simulation show that the perpendicular component of the magnetization is deflected by 0.04 , where 1 means a 90-degree deviation from the static configuration. This value is large, especially considering that the magnetization vibrates mainly in the plane. For the $m_{y}$ component, this deviation from the $x$ axis reaches over 0.3 (cf. SM [22], Figs. S5 and S7). We observe that all antinodes are spatially in phase, excluding a simple standing SW and reinforcing our interpretation as driven STC. Here, it is important to note that the space periodicity is found at $k_{1}$ and not at $2 k_{1}$, which would be a logical consequence of a simple standing-wave structure. While we observe a perfect STC in simulations [Fig. 3(b)], experimentally, we only detect condensation of a STC in the center of the waveguide [Fig. 3(a)], as one can see within the marked region. In the experiment, slight misalignments of the sample with respect to the external bias field lead to the formation of
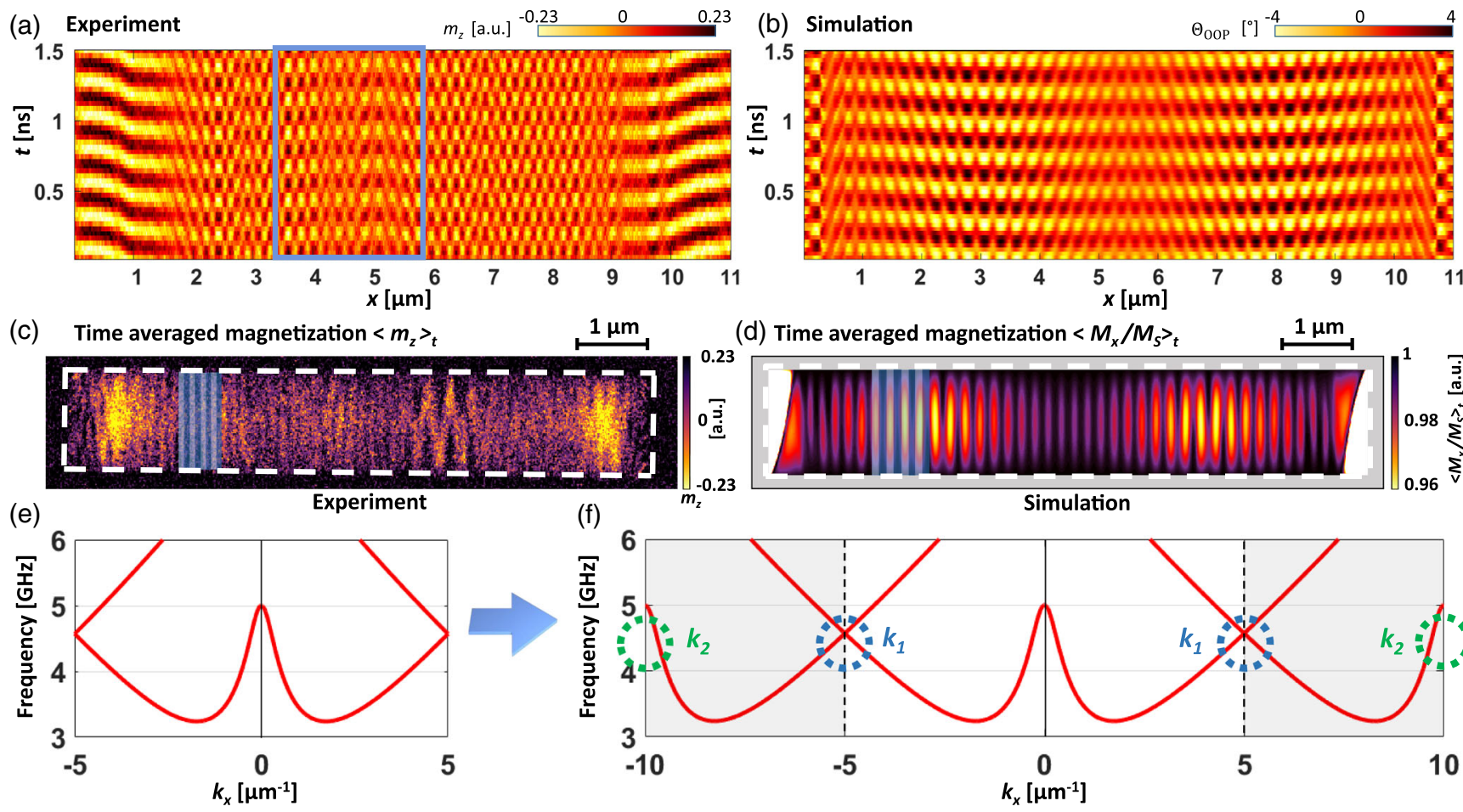

(d) Time averaged magnetization $\left\langle M_{x} / M_{s}\right\rangle_{t}$
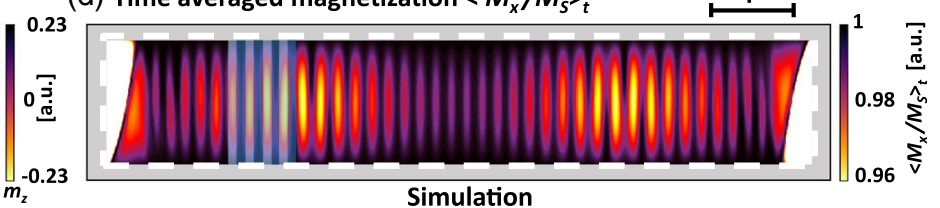

(f)

Simulation
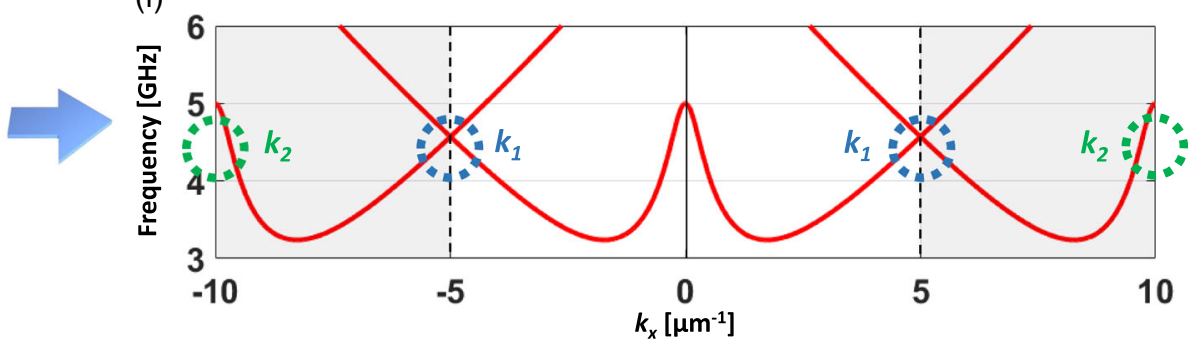

FIG. 3. Spatiotemporal evolution of the $m_{z}$ component of the magnetization from the experiment (a) and the angle $\theta_{\mathrm{OOP}}=\arcsin \left(m_{z}\right)$ made by the magnetization with the $x$ axis along the out-of-plane direction from a simulation (b). The results show good qualitative agreement of a periodic magnetization pattern that is spatially in phase, which indicates condensation of a STC. In simulation, all antinodes are spatially in phase, excluding a simple standing SW that would show a checkerboardlike pattern. In experiment, the STC signature is only observed in the center due to a slight misalignment with respect to the external field (marked area). (c) Experimental and (d) micromagnetic landscape of the static magnetization of the STC under microwave excitation, revealing a spatial periodicity of $200 \mathrm{~nm}$ (shown in blue). The high amplitude of the periodic magnetization pattern results in an alternating demagnetization along the bias field direction. (e) Dispersion relation $f\left(k_{x}\right)$ considering the spatial periodicity of $200 \mathrm{~nm}$ that defines the Brillouin zone. Hence, mode folding occurs at $k_{1}=5 \mu \mathrm{m}^{-1}$. Thereby, a (f) band structure is formed that allows $\mathrm{SW}$ excitations at high $k$ vectors, like $k_{2}=10 \mu \mathrm{m}^{-1}$ shown in Fig. 2. 
standing SW signatures at the edges. The detection of a STC in the center, albeit this misalignment, hints at the robustness of STC formation. It is noteworthy that the periodic magnetization pattern expands into the waveguide - first, in a different way (from the sides to the center, instead of from the center to the sides) and, second, at a faster speed than would be expected from the phase velocity of the SWs and their interference (cf. SM [22], Fig. S8). It confirms the nonlinear origin of the STC and further excludes an interference effect and thus a standing SW which can be excited within a confined geometry with a Dzyaloshinskii-Moriya interaction (DMI) and an induced nonreciprocity as reported in Ref. [30]. The present system exhibits neither DMI nor a broken reciprocity. Hence, the periodic magnetization pattern can be considered as driven STC with the SW's wavelength as spatial periodicity $\left(\lambda_{1}=200 \mathrm{~nm}\right)$ and a temporal period equal to the driving frequency.

In contrast to quantum systems, like quantum gases or BECs, we do not experimentally confirm spontaneous TTSB and subharmonic oscillations, although spontaneous TTSB might occur due to stochastic fluctuations caused by Suhl instabilities at high power levels [31]. However, the system presented here still shows spontaneous space symmetry breaking and poses the same periodic modulation in time and space as these STCs. Such a TTSB and, accordingly, a STC are forbidden in thermodynamic equilibrium [11]. In contrast, the STC's ground state is a flux equilibrium [19]. Here, this flux equilibrium $d D / d t=0$ of the magnon density $D$ is achieved by continuous creation of magnons by the driving field and damping of magnons. Indeed, our simulations show that nonzero damping and sufficient pumping power levels, as discussed later, are crucial for the formation of a driven STC. As a result, the emergence of the space-time periodic texture can be categorized as a subgroup of the conventional established STC structures, which is more focused on the impact of the space-time periodicity, e.g., the generation of a band structure and self-scattering processes, than on the formation phase of a STC itself.

To gain further insight into the formation of the STC, a micromagnetic simulation of a simplified system is performed (cf. SM [22], Fig. S9). Therefore, a narrower (200 nm wide) but infinitely long Py waveguide is simulated in a spatially uniform rf field at a frequency near the FMR. We find that for high amplitudes of the rf field, the precession within the whole sample is uniform. However, a small perturbation, like minor variations of the rf field on the order of $10 \mathrm{~nm}$, leads to the creation of a periodic magnetization pattern equivalent to the one measured experimentally. Thus, it is possible that such an extremely small perturbation of the rf field causes an injection of large $k$ vectors. In turn, these magnetization patterns lead to a change of the system's properties so that they are similar to spontaneous translational symmetry breaking.
Above a critical driving power, the high amplitude of the periodic magnetization pattern leads to a demagnetizing effect, reducing the magnetization along the in-plane bias field [32]. This result is shown experimentally and from micromagnetic simulations in Figs. 3(c) and 3(d), respectively, and elaborated on in the SM [22], Fig. S3. Through this imprinting of a modulation of the in-plane magnetization, the STC effectively acts as a magnonic crystal that forms a band structure for SWs [6,33]. As a comparison to the necessity of high power levels, Fig. S4 in the SM [22] shows a phase and amplitude map below the critical power threshold. No formation of the periodic magnetization pattern can be observed. Only the SW modes predicted theoretically by the linear dispersion relation are visible. The high input power in this study constitutes a necessary condition to observe space-time periodic effects but does not serve as a variable parameter.

The first Brillouin zone of this magnonic STC is shown in Fig. 3(e). The zone boundary is given by the fundamental SW vector $k_{1}=5 \mu \mathrm{m}^{-1}$ at the driving frequency $f_{\mathrm{cw}}=4.2 \mathrm{GHz}$. However, as the STC extends over the full sample, higher Brillouin zones and folding of the modes at zone boundaries also need to be considered. The resulting extended band structure is shown in Fig. 3(f), and it becomes evident that higher $k$ modes are also allowed. Figures 4(a) and 4(b) show the observation of such a mode at $k_{2}=10 \mu \mathrm{m}^{-1}$ from experiment and simulation, respectively. While the field and frequency were varied experimentally, this behavior is only observed for this specific resonance condition, suggesting that the nucleation of a STC is a prerequisite for the emergence of SWs at $k_{2}$.

Magnons with $k_{2}=10 \mu \mathrm{m}^{-1}$ are generated by scattering on the periodic magnetization pattern at $k_{1}$ within the STC. It is noteworthy that this pattern at $k_{1}=5 \mu \mathrm{m}^{-1}$ forms the magnonic crystal where magnons can scatter. Thus, this is a self-scattering process, and mode $k_{2}$ in the second Brillouin zone is only significantly populated at large magnonic densities. However, this scattering process is allowed as both energy and momentum are conserved because the FMR mode also lies close to $f_{\mathrm{cw}}$, which leads to efficient rf

\section{(a) Experiment}

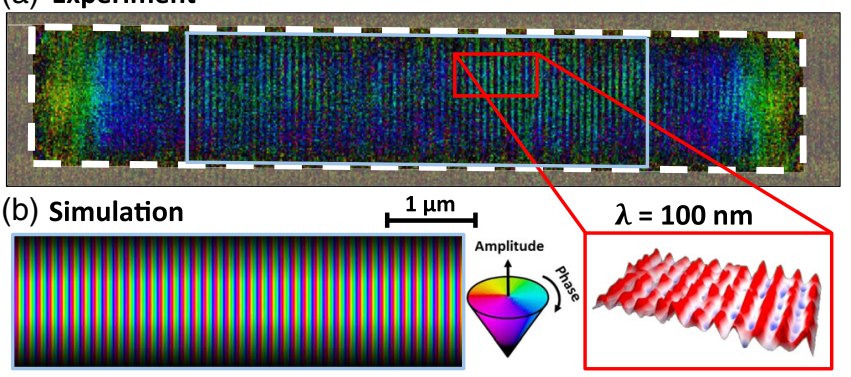

FIG. 4. (a) Experimental phase and amplitude map of mode $k_{2}=10 \mu \mathrm{m}^{-1}$, corresponding to a SW wavelength of $100 \mathrm{~nm}$. The inset illustrates an enlarged area of the mode profile. (b) Comparison of mode $k_{2}$ from micromagnetic simulations. 
field absorption in the form of uniform in-phase magnetization precession serving as a $k \approx 0$ magnon reservoir. It has been confirmed by micromagnetic simulations that the FMR mode as an energy and momentum reservoir is a requirement for efficient STC generation, scattering, and population of $k_{2}$ (cf. SM [22], Fig. S3). In principle, this procedure can be considered as a four-magnon scattering process, yet one magnon is provided by the STC lattice [34]. Thus, this process can also be considered as lattice scattering of a magnon, where the loss of STC magnons into the FMR is compensated by the continuous pumping.

To exclude the mere presence of a Suhl instability, we also perform the experiment and simulations at excitation frequencies below or above the FMR frequency. In these cases, high $k$ modes originating from Suhl instabilities should still emerge at large power levels because the Suhl instability does not rely on the FMR as a momentum reservoir for scattering. However, we do not observe the periodic magnetization pattern or the additional mode $k_{2}$ (cf. SM [22], Fig. S3) in these conditions. Nevertheless, the broadening of the FMR facilitates meeting the resonance condition to create a driven STC. From a theoretical point of view, it is obvious that space-time crystallinity can only exist near the FMR. However, the experimental confirmation shows the validity of our analysis and provides a robust foundation for further experimental studies of interactions with a STC.

In conclusion, we have directly observed a driven STC and the formation of its magnonic band structure in a Py waveguide experimentally by STXM and by micromagnetic simulations. The room-temperature STC is formed by a periodic magnetization pattern that also leads to dynamic demagnetization at nonlinear power levels. Furthermore, we have shown the interaction of quasiparticles with such a STC as we observed in lattice scattering of SWs. Folding of the dispersion relation at the STC Brillouin zone boundaries results in a rich band structure. Thus, lattice scattering processes result in the generation of ultrashort SWs beyond the classical dispersion relation and are observed down to $100 \mathrm{~nm}$.

As driven magnonic STCs can be easily manipulated, this is a unique route to reconfigure magnonic crystals without the need for nanoscale patterning $[4,6]$. Furthermore, the reconfigurable STC band structure allows for efficient SW generation at ultrashort length scales, well below the limits of the classical dispersion relation. However, the observation of interactions with a STC is even more intriguing and is readily accessible by STXM and micromagnetics. In general, we have shown that STCs form band structures at room temperature and that quasiparticles interact with these lattices like in regular crystals, thus promising outstanding new opportunities in fundamental research in nonlinear wave physics.

The authors would like to thank Michael Bechtel for support during beamtimes. We thank Helmholtz-Zentrum
Berlin (HZB) for the allocation of synchrotron beamtime. The research has received partially funding from the Polish National Science Centre project No. 2018/30/Q/ST3/00416 and from the National Science Centre, Poland, Project No. 2019/35/D/ST3/03729. The simulations were partially performed at the Poznan Supercomputing and Networking Center (Grant No. 398). N. T., F. G., M. W., G. S., and J.G. acknowledge the financial support by the Federal Ministry of Education and Research of Germany in the frame work of DynaMAX (Project No. 05K18EYA).

*traeger@is.mpg.de graefe@is.mpg.de

[1] A. V. Chumak, V. I. Vasyuchka, A. A. Serga, and B. Hillebrands, Magnon spintronics, Nat. Phys. 11, 453 (2015).

[2] S. Wintz, V. Tiberkevich, M. Weigand, J. Raabe, J. Lindner, A. Erbe, A. Slavin, and J. Fassbender, Magnetic vortex cores as tunable spin-wave emitters, Nat. Nanotechnol. 11, 948 (2016).

[3] A. V. Chumak, A. A. Serga, and B. Hillebrands, Magnon transistor for all-magnon data processing, Nat. Commun. 5, 4700 (2014).

[4] A. V. Chumak, A. A. Serga, and B. Hillebrands, Magnonic crystals for data processing, J. Phys. D 50, 244001 (2017).

[5] A. V. Sadovnikov, E. N. Beginin, M. A. Morozova, Y. P. Sharaevskii, S. V. Grishin, S. E. Sheshukova, and S. A. Nikitov, Nonlinear spin wave coupling in adjacent magnonic crystals, Appl. Phys. Lett. 109, 042407 (2016).

[6] M. Krawczyk and D. Grundler, Review and prospects of magnonic crystals and devices with reprogrammable band structure, J. Phys. Condens. Matter 26, 123202 (2014).

[7] F. Ma, Y. Zhou, H. B. Braun, and W. S. Lew, Skyrmionbased dynamic magnonic crystal, Nano Lett. 15, 4029 (2015).

[8] F. Wilczek, Quantum Time Crystals, Phys. Rev. Lett. 109, 160401 (2012).

[9] V. Khemani, R. Moessner, and S. L. Sondhi, A brief history of time crystals, arXiv:1910.10745.

[10] H. Watanabe and M. Oshikawa, Absence of Quantum Time Crystals, Phys. Rev. Lett. 114, 251603 (2015).

[11] P. Bruno, Comment on Quantum Time Crystals, Phys. Rev. Lett. 110, 118901 (2013).

[12] V. K. Kozin and O. Kyriienko, Quantum Time Crystals from Hamiltonians with Long-Range Interactions, Phys. Rev. Lett. 123, 210602 (2019).

[13] C. W. von Keyserlingk, V. Khemani, and S. L. Sondhi, Absolute stability and spatiotemporal long-range order in floquet systems, Phys. Rev. B 94, 085112 (2016).

[14] D. V. Else, B. Bauer, and C. Nayak, Floquet Time Crystals, Phys. Rev. Lett. 117, 090402 (2016).

[15] S. Choi, J. Choi, R. Landig, G. Kucsko, H. Zhou, J. Isoya, F. Jelezko, S. Onoda, H. Sumiya, V. Khemani, C. von Keyserlingk, N. Y. Yao, E. Demler, and M. D. Lukin, Observation of discrete time-crystalline order in a disordered dipolar many-body system, Nature (London) 543, 221 (2017). 
[16] J. Zhang, P. W. Hess, A. Kyprianidis, P. Becker, A. Lee, J. Smith, G. Pagano, I. D. Potirniche, A. C. Potter, A. Vishwanath, N. Y. Yao, and C. Monroe, Observation of a discrete time crystal, Nature (London) 543, 217 (2017).

[17] J. Rovny, R. L. Blum, and S. E. Barrett, Observation of Discrete-Time-Crystal Signatures in an Ordered Dipolar Many-Body System, Phys. Rev. Lett. 120, 180603 (2018).

[18] J. Smits, L. Liao, H. T. C. Stoof, and P. van der Straten, Observation of a Space-Time Crystal in a Superfluid Quantum Gas, Phys. Rev. Lett. 121, 185301 (2018).

[19] A. J. E. Kreil, H. Y. Musiienko-Shmarova, S. Eggert, A. A. Serga, B. Hillebrands, D. A. Bozhko, A. Pomyalov, and V.S. L'vov, Tunable space-time crystal in room-temperature magnetodielectrics, Phys. Rev. B 100, 020406(R) (2019).

[20] J. Gräfe, M. Weigand, B. V. Waeyenberge, A. Gangwar, F. Groß, F. Lisiecki, J. Rychly, H. Stoll, N. Träger, J. Förster, F. Stobiecki, J. Dubowik, J. Klos, M. Krawczyk, C. H. Back, E. J. Goering, and G. Schütz, Visualizing Nanoscale Spin Waves Using MAXYMUS, Vol. 11090 of SPIE Nanoscience +Engineering (SPIE, 2019), https://doi.org/10.1117/12 .2530326 .

[21] A. Vansteenkiste, J. Leliaert, M. Dvornik, M. Helsen, F. Garcia-Sanchez, and B. V. Waeyenberge, The design and verification of MuMax3, AIP Adv. 4, 107133 (2014).

[22] See Supplemental Material at http://link.aps.org/supplemental/ 10.1103/PhysRevLett.126.057201 for a detailed application of micromagnetic simulations to carefully model the experiment and to gain a fundamental understanding of the experimental results.

[23] B. Van De Wiele, S. J. Hämälaïnen, P. Baláz, F. Montoncello, and S. Van Dijken, Tunable short-wavelength spin wave excitation from pinned magnetic domain walls, Sci. Rep. 6, 21330 (2016).

[24] F. B. Mushenok, R. Dost, C. S. Davies, D. A. Allwood, B. J. Inkson, G. Hrkac, and V. V. Kruglyak, Broadband conversion of microwaves into propagating spin waves in patterned magnetic structures, Appl. Phys. Lett. 111, 042404 (2017).

[25] N. J. Whitehead, S. A. R. Horsley, T. G. Philbin, A. N. Kuchko, and V. V. Kruglyak, Theory of linear spin wave emission from a Bloch domain wall, Phys. Rev. B 96, 064415 (2017).

[26] F. Groß, N. Träger, J. Förster, M. Weigand, G. Schütz, and J. Gräfe, Nanoscale detection of spin wave deflection angles in permalloy, Appl. Phys. Lett. 114, 012406 (2019).

[27] T. Brächer, P. Pirro, and B. Hillebrands, Parallel pumping for magnon spintronics: Amplification and manipulation of magnon spin currents on the micron-scale, Phys. Rep. 699, 1 (2017).

[28] K. Y. Guslienko, S. O. Demokritov, B. Hillebrands, and A. N. Slavin, Effective dipolar boundary conditions for dynamic magnetization in thin magnetic stripes, Phys. Rev. B 66, 132402 (2002).

[29] B. A. Kalinikos and A. N. Slavin, Theory of dipoleexchange spin wave spectrum for ferromagnetic films with mixed exchange boundary conditions, J. Phys. C 19, 7013 (1986).

[30] B. W. Zingsem, M. Farle, R. L. Stamps, and R. E. Camley, Unusual nature of confined modes in a chiral system: Directional transport in standing waves, Phys. Rev. B 99, 214429 (2019).

[31] H. Suhl, The theory of ferromagnetic resonance at high signal powers, J. Phys. Chem. Solids 1, 209 (1957).

[32] F. Lisiecki, J. Rychły, P. Kuświk, H. Głowiński, J. W. Kłos, F. Groß, N. Träger, I. Bykova, M. Weigand, M. Zelent, E. J. Goering, G. Schütz, M. Krawczyk, F. Stobiecki, J. Dubowik, and J. Gräfe, Magnons in a Quasicrystal: Propagation, Extinction, and Localization of Spin Waves in Fibonacci Structures, Phys. Rev. Applied 11, 054061 (2019).

[33] P. Gruszecki, C. Banerjee, M. Mruczkiewicz, O. Hellwig, A. Barman, and M. Krawczyk, in Chapter Two-The Influence of the Internal Domain Wall Structure on Spin Wave Band Structure in Periodic Magnetic Stripe Domain Patterns (Academic Press, New York, 2019), Vol. 70, p. 79.

[34] H. Schultheiss, K. Vogt, and B. Hillebrands, Direct observation of nonlinear four-magnon scattering in spin-wave microconduits, Phys. Rev. B 86, 054414 (2012). 